University of Nebraska - Lincoln

DigitalCommons@University of Nebraska - Lincoln

3-15-2012

\title{
Resonant phenomena in laser-assisted radiative attachment or recombination
}

A. N. Zheltukhin

Voronezh State University, Voronezh, Russia

A. V. Flegel

University of Nebraska-Lincoln, aflegel6@unl.edu

M. V. Frolov

Voronezh State University, Russia, frolov@phys.vsu.ru

N. L. Manakov

Voronezh State University, Russia, manakov@phys.vsu.ru

Anthony F. Starace

University of Nebraska-Lincoln, astarace1@unl.edu

Follow this and additional works at: https://digitalcommons.unl.edu/physicsstarace

Part of the Physics Commons

Zheltukhin, A. N.; Flegel, A. V.; Frolov, M. V.; Manakov, N. L.; and Starace, Anthony F., "Resonant phenomena in laser-assisted radiative attachment or recombination" (2012). Anthony F. Starace Publications. 189.

https://digitalcommons.unl.edu/physicsstarace/189

This Article is brought to you for free and open access by the Research Papers in Physics and Astronomy at DigitalCommons@University of Nebraska - Lincoln. It has been accepted for inclusion in Anthony F. Starace Publications by an authorized administrator of DigitalCommons@University of Nebraska - Lincoln. 


\title{
Resonant phenomena in laser-assisted radiative attachment or recombination
}

\author{
A. N. Zheltukhin, ${ }^{1}$ A. V. Flegel, ${ }^{2,3}$ M. V. Frolov, ${ }^{1}$ N. L. Manakov, ${ }^{1}$ and Anthony F. Starace ${ }^{2}$ \\ 1. Department of Physics, Voronezh State University, Voronezh, Russia \\ 2. Department of Physics and Astronomy, University of Nebraska-Lincoln, Lincoln, NE, USA \\ 3. Department of Computer Science, Voronezh State University, Voronezh, Russia
}

Corresponding author - A. V. Flegel, email aflegel6@unl.edu

\begin{abstract}
Resonant enhancements are predicted in cross sections $\sigma_{n}$ for laser-assisted radiative attachment or electron-ion recombination accompanied by absorption of $n$ laser photons. These enhancements occur for incoming electron energies at which the electron can be attached or recombined by emitting $\mu$ laser photons followed by emission of a spontaneous photon upon absorbing $n+\mu$ laser photons. The close similarity between rescattering plateaus in spectra of resonant attachment/recombination and of high-order harmonic generation is shown based on a general parametrization for $\sigma_{n}$ and on numerical results for $e-H$ attachment.
\end{abstract}

An electron colliding with an atom in the presence of an intense laser field can efficiently convert the combined energy of a large number of laser photons into the energy of a spontaneously emitted photon [1]. This process occurs, in particular, in laser-assisted radiative attachment (LARA) or recombination (LARR), in which the emission of a spontaneous photon is accompanied by absorption of $n$ laser photons and the formation of a negative ion or neutral atom. Although experimental studies of these processes have only begun recently [2], theoretical studies began more than a decade ago. The first theoretical investigations employed the strong field approximation (SFA) [3-5], in which the effects of the atomic potential $U(r)$ on the scattering state of the incident electron are neglected. These studies show that (even in lowest order in the potential $U(r)$ ) the LARR cross sections as a function of $n$ (or the energy of the spontaneous photon) exhibit a plateau structure, whose shape and extent can be described by treating the LARR process classically [6]. The first-order correction in the potential $U(r)$ to the SFA LARR amplitude was introduced in [7] (see also [1,8]) taking into account $U(r)$ perturbatively, using the Born approximation. Inclusion of higher-order corrections in $U(r)$ (or rescattering effects) into the LARA/LARR amplitudes results in a second, high-energy (or rescattering) plateau in the
LARA/LARR spectra $[7,9]$. However, as for laser-induced processes, such as high-order harmonic generation (HHG) and above-threshold ionization (cf [1]), the cross sections for the high energy rescattering plateau are orders ofmagnitude smaller than those for the lowenergy plateau. Hence, mechanisms for increasing the high-energy plateau cross sections are of great interest. For laser-assisted collisions, one way to achieve such an increase is to tune the incoming electron energy so that it can be temporarily captured (by stimulated emission of $\mu$ laser photons) to a bound state of the potential $U(r)$. Obviously, such a resonance phenomenon cannot be described in the Born approximation and requires an accurate account of the potential $U(r)$. Significant enhancement of plateau structures in resonant laser-assisted electron-atom scattering (LAES) was predicted recently [10]. However, resonant phenomena in LARA/LARR processes remain unexplored.

In the present communication, we extend the study of laser-induced resonant phenomena in collision problems to the case of LARA/LARR. We present a general parametrization for the resonant LARA/LARR cross sections and show the following at resonant electron energies: (i) the shape of LARA/LARR spectra as a function of $n$ coincides with that for HHG; (ii) the electron energy dependence of the n-photon LARA/ 
LARR cross section exhibits an asymmetric profile similar to the Fano profile in photoionization cross sections [11]; and (iii) LARA cross sections can be enhanced by more than two orders of magnitude. To describe LARA analytically, we use time-dependent effective range (TDER) theory, which provides a means to account for the short-range atomic potential $U(r)$ in LARA non-perturbatively.

To describe electron-atom collisions in a monochromatic field with electric vector $\mathbf{F}(t)=\mathbf{e}_{z} F \cos \omega t$ (where $F$ and $\omega$ are the field amplitude and frequency) using the electric dipole approximation, the quasienergy (or Floquet) approach [12] is most appropriate. Within this approach, the laser-dressed scattering state of an electron with momentum $\mathbf{p}$ and energy $E=p^{2} /(2 m)$ in the potential $U(r)$ has the form

$$
\begin{aligned}
& \Psi_{\epsilon, \mathbf{p}}(\mathbf{r}, t)=\mathrm{e}^{-\mathrm{i} \epsilon t / \hbar} \Phi_{\epsilon, \mathbf{p}}(\mathbf{r}, t), \\
& \Phi_{\epsilon, \mathbf{p}}(\mathbf{r}, t)=\Phi_{\epsilon, \mathbf{p}}(\mathbf{r}, t+T), \quad T=2 \pi / \omega,
\end{aligned}
$$

where $\epsilon$ is the quasienergy, $\epsilon=E+u_{p}$, where $u_{p}=\mathrm{e}^{2} F^{2} /$ $\left(4 m \omega^{2}\right)$ is the mean quiver energy of an electron in the field $\mathbf{F}(t)$. For $\mathbf{F}(t)=0$, the quasienergy state $\Phi_{\epsilon, \mathbf{p}}(\mathbf{r}, t)$ reduces to the scattering state $\psi_{\mathrm{p}}(\mathbf{r})$ of the recombining electron in the potential $U(r)$. In the field $\mathbf{F}(t)$, the bound (final) state $\psi_{0}(\mathbf{r})$ with energy $E_{0}$ evolves to the quasistationary quasienergy state (QQES), $\Psi_{\varepsilon}(\mathbf{r}, t)$, which also has the form (1), but with the complex quasienergy $\varepsilon=$ $E_{0}+\Delta E_{0}-\mathrm{i} \Gamma / 2$, where $\Delta E_{0}$ and $\Gamma$ are the field-induced Stark-shift and width (or total decay rate $\Gamma / \hbar$ ) of the state $\psi_{0}(\mathbf{r})[13]$.

We consider the LARA/LARR process as a dipole transition between initial and final states $\Psi_{\epsilon, \mathbf{p}}(\mathbf{r}, t)$ and $\Psi_{\varepsilon}(\mathbf{r}, t)$ with emission of a spontaneous photon, whose energy differs from the field-free energy $\hbar \Omega_{0}=E-E_{0}$. Within the QQES approach, the LARA/LARR cross section $\sigma(\Omega)$, integrated over the directions of emission and summed over polarizations of the spontaneous photon, can be written as (cf [7])

$$
\begin{gathered}
\sigma(\Omega)=\frac{4 m \Omega^{3}}{3 \hbar c^{3} p}|\mathbf{d}(\Omega)|^{2}, \\
\mathbf{d}(\Omega)=\frac{1}{T} \int_{0}^{T} \mathrm{~d} t \int \mathrm{d} \mathbf{r} \tilde{\Psi}_{\varepsilon}^{*}(\mathbf{r}, t) \mathbf{d} \Psi_{\epsilon, \mathbf{p}}(\mathbf{r}, t) \mathrm{e}^{\mathrm{i} \Omega t},
\end{gathered}
$$

where $\mathbf{d}=e \mathbf{r}(e=-|e|)$ and $\Omega$ is the frequency of the spontaneously emitted photon:

$$
\hbar \Omega=\epsilon+n \hbar \omega-\operatorname{Re} \varepsilon .
$$

The function $\tilde{\Psi}_{\varepsilon}(\mathbf{r}, t)$ in (3) is the so-called dual function to $\Psi_{\varepsilon}(\mathbf{r}, t)$. If $\mathbf{F}(t)$ is linearly polarized and $\psi_{0}(\mathbf{r})$ is a bound s-state, then $\Psi_{\varepsilon}(\mathbf{r}, t)$ is defined as [14-16]

$$
\tilde{\Psi}_{\varepsilon}(\mathbf{r}, t)=\mathrm{e}^{-\mathrm{i} \varepsilon^{*} t / \hbar} \Phi_{\varepsilon}^{*}(\mathbf{r},-t)
$$

Since the QQES wavefunctions $\Phi_{\varepsilon}(\mathbf{r}, t)$ diverge asymptotically as $r \rightarrow \infty$ (since they describe the ionization of a bound state $\psi_{0}(\mathbf{r})$ in the field $\left.\mathbf{F}(t)\right)$, the use of dual functions as bra-vectors in the QQES approach is necessary to ensure proper normalization of the wavefunctions $\Phi_{\varepsilon}$ $(\mathbf{r}, t)$ and the regularization of matrix elements involving these functions (cf [14-16] for further details).
To describe resonant LARR or LARA processes, we note first that the wavefunction $\Psi_{\varepsilon}(\mathbf{r}, t)$ can be obtained as a residue of the scattering state $\Psi_{\epsilon, \mathbf{p}}(\mathbf{r}, t)$ in the complex plane of $\epsilon$ at $\epsilon=\varepsilon+\mu \hbar \omega=\operatorname{Re} \varepsilon+\mu \hbar \omega-\mathrm{i} \Gamma / 2$ [17]:

$$
\left.\operatorname{Res} \Psi_{\epsilon, \mathbf{p}}(\mathbf{r}, t)\right|_{\epsilon=\varepsilon+\mu \hbar \omega} \sim \mathrm{e}^{\mathrm{i} \mu \omega t} \Psi_{\varepsilon+\mu \hbar \omega}(\mathbf{r}, t)=\Psi_{\varepsilon}(\mathbf{r}, t),
$$

where $\mu$ is an integer. Therefore, for $\epsilon \approx \epsilon_{\mu}=\operatorname{Re} \varepsilon+\mu \hbar \omega$, the scattering state $\Phi_{\epsilon, \mathrm{p}}(\mathbf{r}, t)$ can be approximated by a sum of potential (non-resonant) and resonant parts [17]:

$$
\Phi_{\epsilon, \mathbf{p}}(\mathbf{r}, t)=\Phi_{\epsilon_{\mu}, \mathbf{p}_{\mu}}^{(p)}(\mathbf{r}, t)+\mathcal{B}\left(\mathbf{p}_{\mu}\right) \frac{\Phi_{\varepsilon}(\mathbf{r}, t)}{E-E_{\mu}+\mathrm{i} \Gamma / 2},
$$

where $E_{\mu}=\mathbf{p}^{2}{ }_{\mu} /(2 m)=\operatorname{Re} \varepsilon+\mu \hbar \omega-u_{p}$ is the resonant electron energy and the coefficient $\mathcal{B}\left(\mathbf{p}_{\mu}{ }_{\mu}\right)$ is proportional to the amplitude for stimulated $\mu$-photon recombination or attachment (cf (30)). Substituting (5) into (3), the amplitude $\mathbf{d}(\Omega)$ can also be presented as a sum of potential and resonant terms:

$$
\mathbf{d}(\Omega)=\mathbf{d}^{(p)}(\Omega)+\mathcal{B}\left(\mathbf{p}_{\mu}\right) \frac{\tilde{\mathbf{d}}(\Omega)}{E-E_{\mu}+\mathrm{i} \Gamma / 2},
$$

where the potential term $\mathbf{d}^{(p)}(\Omega)$ is given by (3) (upon substituting there $\Phi^{(p)}{ }_{\mu, \mathbf{p}_{\mu}}(\mathbf{r}, t)$ for $\left.\Phi_{\epsilon, \mathbf{p}}(\mathbf{r}, t)\right)$, while the resonant term involves the dual dipole moment, $\tilde{\mathrm{d}}(\Omega)=$ $\tilde{d}(\Omega) \mathbf{e}_{z}$, which determines the rate $\mathcal{R}(\Omega)$ for the generation of a harmonic of the field $\mathbf{F}(t)$ with frequency $\Omega=(n$ $+\mu) \omega$ by a bound electron in the $s$-state $\psi_{0}(\mathbf{r})$ [16]:

$$
\mathcal{R}(\Omega)=\frac{\Omega^{3}}{2 \pi \hbar c^{3}}|\tilde{\mathbf{d}}(\Omega)|^{2} .
$$

Since the problem involves only two vectors, $\mathbf{e}_{z}$ and $\hat{\mathbf{p}}$ (mutually oriented at an angle $\theta$ ), the vector $\mathbf{d}^{(p)}(\Omega)$ lies in the plane $\left(\mathbf{e}_{z^{\prime}} \hat{\mathbf{p}}\right)$ and can be presented as

$$
\mathbf{d}^{(p)}(\Omega)=d_{\|}^{(p)}(\Omega) \mathbf{e}_{z}+d_{\perp}^{(p)}(\Omega) \mathbf{e}_{\perp},
$$

where $\mathbf{e}_{\perp}=\left[\mathbf{e}_{z} \times\left[\hat{\mathbf{p}} \times \mathbf{e}_{z}\right]\right]$. Using (6) and (8), we obtain the general parametrization for the LARA/LARR cross section (2) near a $\mu$-photon resonance

$$
\sigma(\Omega)=\sigma^{(p)}(\Omega)+\sigma_{\|}^{(p)}(\Omega) \frac{2(\operatorname{Re} q-\delta \operatorname{Im} q)+|q|^{2}}{\delta^{2}+1},
$$

where $\delta=2\left(E-E_{\mu}\right) / \Gamma, q=-2 \mathrm{i}\left(\mathbf{p}_{\mu}\right) \tilde{d}(\Omega) /\left(\Gamma d_{\|}^{(p)}(\Omega)\right.$, and $\sigma^{(p)}(\Omega)$ and $\sigma_{\|}^{(p)}(\Omega)$ are given by $(2)$ upon substituting there $\mathbf{d}(\Omega) \rightarrow \mathbf{d}^{(p)}(\Omega)$ or $\mathbf{d}(\Omega) \rightarrow d_{\|}^{(p)}(\Omega) \mathbf{e}_{z}$. The parametrization (9) simplifies for parallel geometry, $\mathbf{p} \| \mathbf{e}_{z^{\prime}}$ in which case $\sigma_{\|}^{(p)}=\sigma^{(p)}$ :

$$
\sigma(\Omega)=\sigma^{(p)}(\Omega) \frac{(\operatorname{Re} q+1)^{2}+(\operatorname{Im} q-\delta)^{2}}{\delta^{2}+1} .
$$

Results (9) and (10) showthat (for a given $n) \sigma(\Omega)$ as a function of $E$ is asymmetric with respect to the resonance energy $E_{\mu}$. For small $\Gamma$ (i.e. taking into account only terms $\sim 1 / \Gamma^{2}$ ), the result for the cross section $\sigma(\Omega)$ at the resonance, $\delta=0$, is

$$
\sigma(\Omega) \approx \frac{32 \pi m\left|\mathcal{B}\left(\mathbf{p}_{\mu}\right)\right|^{2}}{3 p_{\mu} \Gamma^{2}} \mathcal{R}(\Omega) .
$$

Since $\mathcal{B}\left(\mathbf{p}_{\mu}\right)$ does not depend on the number of absorbed photons, the shapes of resonant LARA/LARR spectra as 
functions of $n$ replicate the shapes of the corresponding bound state HHG spectra.

To present quantitative results for laser-induced resonance phenomena in LARA, we use TDER theory to describe both the incident continuum $\left(\Phi_{\epsilon, \mathrm{p}}\right)[18]$ and final bound $\left(\Phi_{\varepsilon)}[19]\right.$ field-dressed states of the active electron. This theory assumes that the interaction of an electron with a short-range potential $U(r)$ (having only a single bound state $\psi_{\text {Eolm }}(\mathbf{r})$ with angular momentum $l$ ) is described by the $l$-wave scattering phase $\delta_{l}(E)$ that is parameterized by the scattering length $a_{l}$ and the effective range $r_{l}$, which are parameters of the problem. For simplicity, we consider the case of a bound s-state $\psi_{0}(\mathbf{r})$ of energy $E_{0}=-(\hbar \kappa)^{2} /(2 m)$. For this case, the TDER wavefunctions $\Phi_{\epsilon, \mathbf{p}}(\mathbf{r}, t)$ and $\Phi_{\varepsilon}(\mathbf{r}, t)$ are expressed in terms of one-dimensional integrals $[18,19]$ :

$$
\begin{gathered}
\Phi_{\epsilon, \mathbf{p}}(\mathbf{r}, t)=\mathrm{e}^{\mathrm{i}[\mathbf{P}(t) \cdot \mathbf{r}-\mathcal{S}(\mathbf{p}, t)] / \hbar} \\
-\frac{2 \pi \hbar^{2}}{m \kappa} \int G\left(\mathbf{r}, t, 0, t^{\prime}\right) f_{\epsilon}\left(\mathbf{p}, t^{\prime}\right) \mathrm{e}^{\mathrm{i} \epsilon\left(t-t^{\prime}\right) / \hbar} \mathrm{d} t^{\prime}, \\
\Phi_{\varepsilon}(\mathbf{r}, t)=-\frac{2 \pi \hbar^{2} \sqrt{\kappa}}{m} \int G\left(\mathbf{r}, t, 0, t^{\prime}\right) g_{\varepsilon}\left(t^{\prime}\right) \mathrm{e}^{\mathrm{i} \varepsilon\left(t-t^{\prime}\right) / \hbar} \mathrm{d} t^{\prime},
\end{gathered}
$$

where $G\left(\mathbf{r}, t, \mathbf{r}^{\prime}, t^{\prime}\right)$ is the retarded Green function and $S(\mathbf{p}$, $t$ ) is the classical action of an electron in the field $\mathbf{F}(t)$,

$$
\mathcal{S}(\mathbf{p}, t)=\int^{t}\left[\mathbf{P}^{2}\left(t^{\prime}\right) /(2 m)-\epsilon\right] \mathrm{d} t^{\prime},
$$

$\mathbf{P}(t)$ is the canonical momentum,

$$
\mathbf{P}(t)=\mathbf{p}-\mathbf{e}_{z} \frac{e F}{\omega} \sin \omega t,
$$

and $f_{\epsilon}(\mathbf{p}, t)$ and $g_{\varepsilon}(t)$ are dimensionless periodic functions,

$$
f_{\epsilon}(\mathbf{p}, t)=\sum_{k} f_{k}(\mathbf{p}) \mathrm{e}^{-\mathrm{i} k \omega t}, \quad g_{\varepsilon}(t)=\sum_{k} g_{k} \mathrm{e}^{-\mathrm{i} k \omega t} .
$$

The Fourier-coefficients $f_{k}(\mathbf{p})$ and $g_{k}$ as well as the complex quasienergy $\varepsilon$ can be found from a system of inhomogeneous (for $f_{k}(\mathbf{p})$ ) or homogeneous (for $g_{k}$ and $\varepsilon$ ) linear equations:

$$
\begin{gathered}
\sum_{k^{\prime}} \mathcal{M}_{k, k^{\prime}}(\epsilon) f_{k^{\prime}}(\mathbf{p})=c_{k}(\mathbf{p}), \\
\sum_{k^{\prime}} \mathcal{M}_{k, k^{\prime}}(\varepsilon) g_{k^{\prime}}=0 \\
\mathcal{M}_{k, k^{\prime}}(\mathcal{E})=R(\mathcal{E}+k \hbar \omega) \delta_{k, k^{\prime}}+M_{k, k^{\prime}}(\mathcal{E}), \\
R(\mathcal{E})=\frac{1}{a_{0} \kappa}-\frac{m r_{0} \mathcal{E}}{\hbar^{2} \kappa}+\mathrm{i} \sqrt{\frac{\mathcal{E}}{\left|E_{0}\right|}} \\
c_{k}(\mathbf{p})=\mathrm{i}^{-k} \sum_{s} J_{k+2 s}\left(\frac{2 e F p_{\|}}{m \hbar \omega^{2}}\right) J_{-s}\left(\frac{u_{p}}{2 \hbar \omega}\right),
\end{gathered}
$$

where $a_{0}$ and $r_{0}$ are the scattering length and the effective range, $p_{\|}=\left(\mathbf{e}_{z} \cdot \mathbf{p}\right)=p \cos \theta$, and $J_{n}(x)$ is a Bessel function. The matrix elements $M_{k, k^{\prime}}(\mathcal{E})$ are nonzero only if the difference $k-k^{\prime}$ is even and have the form

$$
\begin{array}{r}
M_{k, k^{\prime}}(\mathcal{E})=\sqrt{\frac{\mathrm{i}^{k-k^{\prime}} \hbar \omega}{8 \pi \mathrm{i}\left|E_{0}\right|}} \int_{0}^{\infty} \frac{\mathrm{d} \tau}{\tau^{3 / 2}} \mathrm{e}^{\mathrm{i}\left[2 \mathcal{E} /(\hbar \omega)+\left(k+k^{\prime}\right)\right] \tau} \\
\times\left\{\mathrm{e}^{\mathrm{i} \lambda(\tau)} J_{\left(k-k^{\prime}\right) / 2}[z(\tau)]-\delta_{k, k^{\prime}}\right\},
\end{array}
$$

where

$$
\begin{array}{r}
\lambda(\tau)=\frac{2 u_{p}}{\hbar \omega}\left(\frac{\sin ^{2} \tau}{\tau}-\tau\right), \\
z(\tau)=\frac{u_{p}}{\hbar \omega}\left(\sin 2 \tau-\frac{2 \sin ^{2} \tau}{\tau}\right) .
\end{array}
$$

From the explicit form of $M_{k, k^{\prime}}(\mathcal{E})$ follow the symmetry relations

$$
\begin{array}{r}
\mathcal{M}_{k, k^{\prime}}(\mathcal{E})=\mathcal{M}_{k^{\prime}, k}(\mathcal{E}), \\
\mathcal{M}_{k, k^{\prime}}(\mathcal{E}+p \hbar \omega)=\mathcal{M}_{k+p, k^{\prime}+p}(\mathcal{E}) .
\end{array}
$$

As shown in [19], the function $g_{\varepsilon}(t)$ (as well as the system of equations (15)) includes only coefficients $g_{k}$ with even $k$. The complex quasienergy $\varepsilon$ is given by that root of the transcendental equation, Det $\left\|M_{k^{\prime}, k}(\varepsilon)\right\|=0$, which becomes $E_{0}$ when $F \rightarrow 0$. For $\mathbf{F}(t)=0$, the matrix elements $M_{k, k^{\prime}}(\mathcal{E})$ are zero and coefficients $f_{k}(\mathbf{p})$ and $g_{k}$ reduce to

$$
f_{k}(\mathbf{p})=R^{-1}(E) \delta_{k, 0}, \quad g_{k}=C_{\kappa} \delta_{k, 0},
$$

where $C_{\kappa}$ is a dimensionless coefficient in the asymptotic form of $\psi_{0}(\mathbf{r})$ for $r \gg \kappa^{-1}$ :

$$
\psi_{0}(\mathbf{r}) \approx C_{\kappa} \sqrt{\kappa /(4 \pi)} r^{-1} \exp (-\kappa r) .
$$

With the use of (12) and (13), the analytic evaluation of $\mathbf{d}(\Omega)$ in (3) involves the spatial integration of two Green functions and a threefold integration over time. The spatial integration and two of the temporal integrations can be performed analytically (as done in [16]) and the final result for $\mathbf{d}(\Omega)$ can be presented as

$$
\begin{gathered}
\mathbf{d}(\Omega)=\mathbf{d}_{p w}(\Omega)+\mathbf{d}_{\mathrm{resc}}(\Omega), \\
\mathbf{d}_{p w}(\Omega)=\mathcal{C}\left[\mathbf{e}_{z} \sum_{p= \pm 1} \frac{\mathcal{L}_{n+p}}{\omega / \Omega+p}-2 \mathrm{i} \mathcal{L}_{n} \frac{\mathbf{p} \omega}{|e| F}\right], \\
\mathcal{L}_{n}(\mathbf{p})=\sum_{\substack{k=-\infty \\
\mathbf{d}_{\text {resc }}(\Omega)}}^{\infty} g_{k} c_{k+n}(\mathbf{p}), \quad \mathcal{C}=\frac{\pi \mathrm{e}^{2} \hbar F \kappa^{1 / 2}}{m^{2} \omega \Omega^{2}}, \\
\sum_{k, k^{\prime}} f_{k}(\mathbf{p}) W_{k, k^{\prime}}^{n} g_{k^{\prime}},
\end{gathered}
$$

where the matrix elements $W_{k, k^{\prime}}^{n}$ are non-zero only if the difference $n-k$ is odd :

$$
\begin{gathered}
W_{k, k^{\prime}}^{n}=\mathcal{D} \int_{0}^{\infty} \frac{\mathrm{d} \tau}{\tau^{3 / 2}} \mathrm{e}^{\mathrm{i}\left[(\epsilon+\varepsilon) /(\hbar \omega)+k+k^{\prime}\right] \tau+\mathrm{i} \lambda(\tau)} \\
\times\left\{j_{-}(\tau) J_{s}[z(\tau)]-\mathrm{i} j_{+}(\tau) J_{s+1}[z(\tau)]\right\}, \\
j_{ \pm}(\tau)=\frac{\sin \tau \sin (\Omega \tau / \omega)}{\tau}-\frac{\Omega \sin (\Omega / \omega \pm 1) \tau}{1 \pm \omega / \Omega}, \\
s=\frac{n-k+k^{\prime}-1}{2}, \quad \mathcal{D}=\mathrm{i}^{s+1 / 2} \sqrt{\frac{\hbar \omega}{2 \pi\left|E_{0}\right|}}
\end{gathered}
$$

Result (22) for $\mathbf{d}(\Omega)$ is exact within the TDER theory and valid for both resonant and non-resonant electron energies $E$. The term $d_{p w}(\Omega)$ originates from the first term in (12) and corresponds to the first-Born (or plane wave) approximation in the potential $U(r)$ for the scattering state. This term is smooth at the resonant energy $E=E_{\mu}$ and contributes only to the potential part of the 

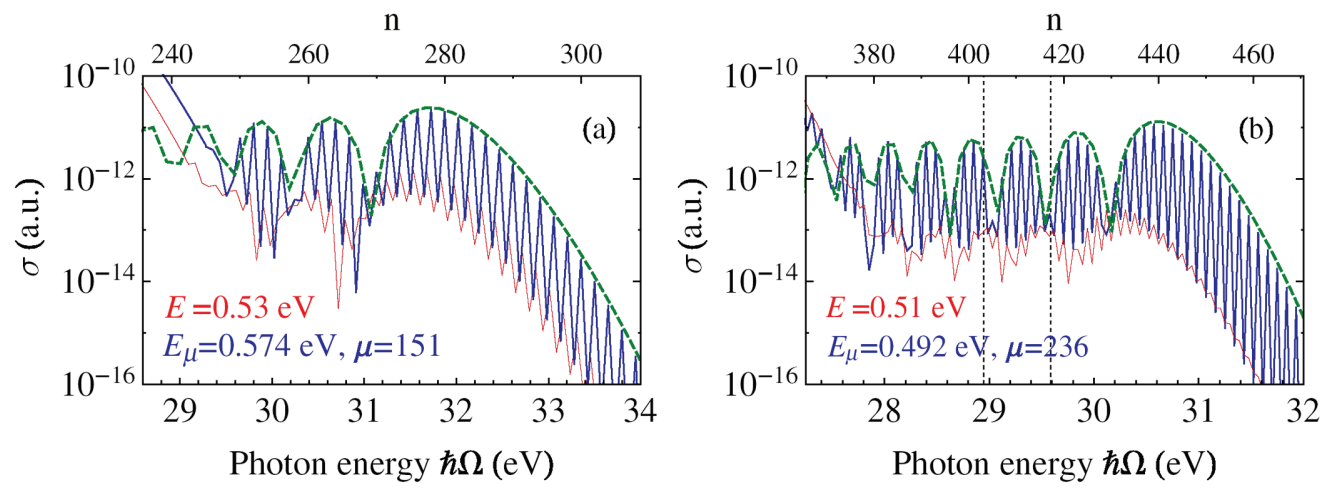

Figure 1. LARA spectra for $e-H$ attachment in a linearly polarized field $\mathbf{F}(t)$ (with $\mathbf{e}_{z} \| \mathbf{p}$ ) having (a) intensity $I=3.75 \times 10^{11} \mathrm{~W}$ $\mathrm{cm}^{-2}, \hbar \omega=0.098\left|E_{0}\right|=0.074 \mathrm{eV}$ or (b) $I=1.35 \times 10^{11} \mathrm{~W} \mathrm{~cm}^{-2}, \hbar \omega=0.06\left|E_{0}\right|=0.0453 \mathrm{eV}$. Thick (blue) and thin (red) solid lines: exact TDER results for the resonant $\left(E_{\mu}\right)$ and non-resonant $(E)$ electron energies shown in each panel. Dashed (green) lines: HHG spectra of the $\mathrm{H}^{-}$ion (in arbitrary units) for the same field parameters $I, \omega$ as in (a) and (b). Vertical lines in (b) mark photon numbers $n=403,417$ (cf figure 2).

LARA amplitude (6). Moreover, for an intense low-frequency ( $\left.\hbar \omega \ll \mid E_{0}\right)$ field $\mathbf{F}(t)$, the coefficients $g_{k}$ with $k$ $=0$ are small compared to $g_{0}$ (cf [19]). Approximating in (24) $g_{k}=g_{0} \delta_{k, 0}$ and $g_{0} \approx C_{k}(\operatorname{cf}(21)), \mathbf{d}_{p w}(\Omega)$ yields an exact (i.e. without using saddle-point methods for its evaluation) TDER result for the LARA amplitude in the SFA $[3,4]$. Resonant phenomena are described by the second (rescattering) term, $\mathbf{d}_{\text {resc }}(\Omega)$, in (22). This term originates from the integral term in (12) and, within the TDER theory, ensures an exact account of the effects of the potential $U(r)$ on the field-dressed initial and final states of the attaching electron (through the coefficients $f_{k}(\mathbf{p})$ and $g_{k}$ in (25)).

To extract from (25) the resonant part of the amplitude (6) in an explicit form, we solve the system (14) for $f_{k}(\mathbf{p})$ near the resonance, i.e. for $\epsilon \sim(\varepsilon+\mu \hbar \omega)$. Expanding matrix elements in (14) up to the linear term in $\Delta \epsilon=\epsilon-\varepsilon-\mu \hbar \omega=E-E_{\mu}+\mathrm{i} \Gamma / 2$, approximating $c_{k}(\mathbf{p}) \approx c_{k}\left(\mathbf{p}_{\mu}\right)$ and employing the symmetry relations (20), we obtain

$$
\begin{aligned}
& \sum_{k^{\prime}} \mathcal{M}_{k, k^{\prime}}(\varepsilon) f_{k^{\prime}-\mu}(\mathbf{p}) \\
& \quad+\Delta \epsilon \sum_{k^{\prime}} \mathcal{M}_{k, k^{\prime}}^{\prime}(\varepsilon) f_{k^{\prime}-\mu}(\mathbf{p})=c_{k-\mu}\left(\mathbf{p}_{\mu}\right),
\end{aligned}
$$

where $M^{\prime}{ }_{k, k^{\prime}}(\varepsilon)=\partial M^{\prime}{ }_{k, k^{\prime}}(\varepsilon) / \partial \varepsilon$. In the lowest resonant approximation $(\Delta \epsilon \rightarrow 0)$, the coefficients $f_{k^{\prime}-\mu}(\mathbf{p})$ in (27) are proportional to $g_{k^{\prime}}: f_{k^{\prime}-\mu}=\alpha(\mathbf{p}) g_{k^{\prime}}$. To find $\alpha(\mathbf{p})$, we multiply the system (27) by $g_{k}$ and then sum over $k$. Taking into account the symmetry relations (20) and the equality $\Sigma_{k} g_{k} M_{k, k^{\prime}}(\varepsilon)=0(\mathrm{cf}(15))$, the system (27) reduces to a single equation

$$
\Delta \epsilon \sum_{k, k^{\prime}} g_{k} \mathcal{M}_{k, k^{\prime}}^{\prime}(\varepsilon) f_{k^{\prime}-\mu}(\mathbf{p})=\sum_{k} g_{k} c_{k-\mu}\left(\mathbf{p}_{\mu}\right),
$$

from which $\alpha(\mathbf{p})$ is easily obtained upon substituting $f_{k^{\prime}-\mu}(\mathbf{p})=\alpha(\mathbf{p}) g_{k^{\prime}}$. The resulting resonant approximation for $f_{k-\mu}(\mathbf{p})$ is

$$
f_{k-\mu}^{(r)}(\mathbf{p})=\frac{g_{k}}{E-E_{\mu}+\mathrm{i} \Gamma / 2} \frac{\sum_{s} g_{s} c_{s-\mu}\left(\mathbf{p}_{\mu}\right)}{\sum_{s, s^{\prime}} g_{s} \mathcal{M}_{s, s^{\prime}}^{\prime}(\varepsilon) g_{s^{\prime}}} .
$$

Changing in (25) the summation index $k$ to $k-\mu$ and substituting there the result (29) for $f_{k-\mu}(\mathbf{p})$, the resonant term in $\mathbf{d}_{\text {resc }}(\Omega)$ can be presented in the same form as in (6), where the explicit form for the dual dipolemoment $\tilde{\mathbf{d}}$ $(\Omega)$ in the TDER theory (in terms of $g_{k^{\prime}} g_{k^{\prime}}$ and the matrix elements $W_{\left.n k-\mu, k^{\prime}\right)}$ is given in [20]. The coefficient $\mathcal{B}\left(\mathbf{p}_{\mu}\right)$ in (6) is related to the amplitude $A_{\mu}\left(\mathbf{p}_{\mu}\right)$ for $\mu$-photon laser-stimulated attachment:

$$
\begin{aligned}
A_{\mu}\left(\mathbf{p}_{\mu}\right) & =\sum_{k} g_{k}^{*} c_{k-\mu}^{*}\left(\mathbf{p}_{\mu}\right), \\
\mathcal{B}\left(\mathbf{p}_{\mu}\right) & =\frac{2 \pi \hbar^{2} \sqrt{\kappa}}{m} A_{\mu}^{*}\left(\mathbf{p}_{\mu}\right) .
\end{aligned}
$$

The potential part $\mathbf{d}^{(p)}(\Omega)$ of $\mathbf{d}(\Omega)$ in (6) is given within TDER theory by (22)-(25) in which we set $\mathbf{p}=\mathbf{p}_{\mu}$ and replace $f_{k}\left(\mathbf{p}_{\mu}\right)$ in (25) by $f_{k}\left(\mathbf{p}_{\mu}\right)-f^{(r)}\left(\mathbf{p}_{\mu}\right)$.

Key features of resonant LARA cross sections are shown in figure 1. (Qualitatively, resonant LARR features are similar.) Results for $e-H$ attachment with the formation of the $\mathrm{H}^{-}$ion are shown for both non-resonant $(E)$ and resonant $\left(E_{\mu}\right)$ incident electron energies for the cases of odd $\mu$ in (a) and even $\mu$ in (b). (TDER parameters for this case are (cf, e.g., [19]) $\left|E_{0}\right|=0.755 \mathrm{eV}, C_{\kappa}=2.304$, $a_{0}=6.16 a_{B}$ and $r_{0}=2.64 a_{B}$, where $a_{B}$ is the Bohr radius.) In Figure 1(a), the laser parameters and energy $E$ are the same as in a recent analysis of nonresonant LARA processes [9]. Resonant effects are more pronounced in figure 1 (b) for $I=1.35 \times 10^{11} \mathrm{~W} \mathrm{~cm}^{-2}$ and $\hbar \omega=0.0453 \mathrm{eV}$. Figure 1 exhibits several qualitative features: (i) a two orders of magnitude resonant increase of $\sigma(\Omega)$; (ii) perfect coincidence of the resonant attachment spectrum shape with that for high harmonics generated by the $\mathrm{H}^{-}$ion for the same laser parameters; (iii) the extent of the highenergy plateau in the resonant process $\left(\approx\left|E_{0}\right|+3.17 u_{p}\right.$ as in HHG) exceeds that for the non-resonant case; and (iv) enhancements occur only for those numbers $n$ of absorbed photons whose parity is opposite to that of $\mu$ (cf also Figure 2). This last result is a simple consequence of the fact that the resonant cross section (11) involves the rate for emission of the $(n+\mu)$ th harmonic of the field $\mathbf{F}(t)$. As is well known, an atom can emit only odd harmonics of a monochromatic field (owing to electric dipole selection rules), so that $n+\mu$ must be odd.

Figure 2 shows the energy dependence of the partial (n-photon) LARA cross sections in the resonance region (as well as our general parametrization for $\sigma_{n}(E)$ ) for the 

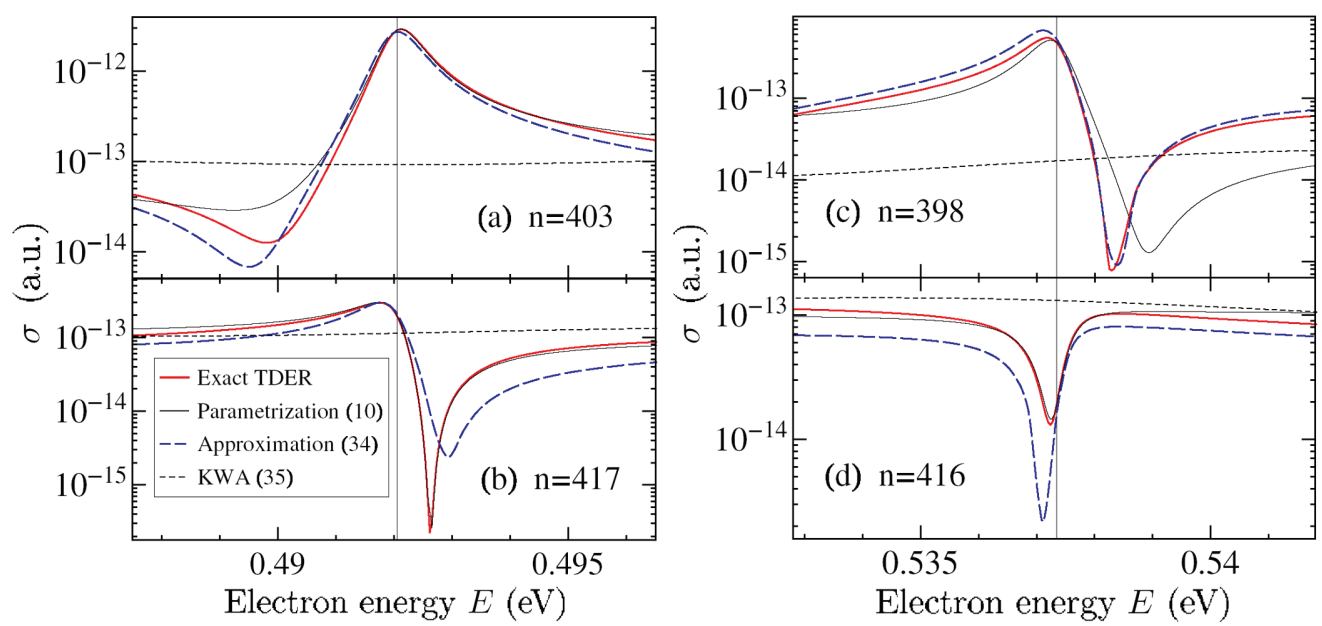

Figure 2. Dependence of LARA cross sections on electron energy for fixed $n$ and the same laser parameters as in Figure 1(b). Thick solid lines: exact TDER results; thin solid lines: parametrization (10); dashed lines: results with coefficients $f_{k}$ given by (34) and $g_{k}=$ $C_{\kappa} \delta_{k^{\prime} 0}$; dotted lines: results with Fourier-coefficients of the KWA function (35). Vertical lines mark the resonance energies $E_{\mu}(\delta=0)$.

laser parameters of Figure 1(b), giving a Stark-shift and width of the $\mathrm{H}^{-}$ground state $\psi_{0}(\mathbf{r})$ of $E_{0}=\operatorname{Re} \varepsilon-E_{0}=$ $-6.250 \times 10^{-3} \mathrm{eV}$ and $\Gamma=8.335 \times 10^{-4} \mathrm{eV}$. Owing to the complexity of the parameter $q$ (i.e. the ratio of the resonant and potential parts of the amplitude (6) at $E=E_{\mu}$ ), the asymmetric resonance profile shape as well as the positions of the maxima and minima in $\sigma_{n}(E)$ (for given $n$ and laser parameters) are sensitive to both the absolute value of $q$ and the relation between $\operatorname{Re} q$ and $\operatorname{Im} q$. Figure 2 shows examples of resonance profiles for both odd $(\mu=236)$ and even $(\mu=237)$ values of $n$. In terms of $\delta$, the positions of the maxima $\left(\delta_{+}\right)$and minima $\left(\delta_{-}\right)$ of $\sigma_{n}(E)$ can be obtained from (9) or (10) by equating to zero the derivative of $\sigma(\Omega)$ with respect to $\delta$ :

$$
\delta_{ \pm}=\frac{|q+1|^{2} \mp|q(q+2)|-1}{2 \operatorname{Im} q} .
$$

Since $\delta_{ \pm}$are the roots of a quadratic equation, the following relations are valid:

$$
\delta_{+} \delta_{-}=-1, \quad \delta_{+}-\delta_{-}=-|q(q+2)| / \operatorname{Im} q .
$$

Relations (32) show that for $\operatorname{Im} q<0$ (or $\operatorname{Im} q>0$ ) the maximum occurs at $E>E_{\mu}$ (or $E<E_{\mu}$ ), while the location of the minimum has the opposite behavior. These results agree with those in Figures 2(a) and (d) (in which $\operatorname{Im} q<0$ ) and 2(b) and (c) (in which $\operatorname{Im} q>0$ ). The "window resonance" behavior of $\sigma_{n}(E)$ (as in Figure 2(d)) occurs whenever $\left|\delta_{-}\right| \rightarrow 0$ or, equivalently, the parameter $\Delta \rightarrow 1$, where

$$
\Delta=\frac{\delta_{+}-\delta_{-}}{\delta_{+}+\delta_{-}}=\frac{|q(q+2)|}{1-|q+1|^{2}} .
$$

The parameter is positive when $|q+1|<1$, and this latter inequality is fulfilled for negative $\operatorname{Re} q($ with $(\operatorname{Re} q)$ $\min \rightarrow-2$ when $|\operatorname{Im} q| \rightarrow 0$ ). The limiting case $\Delta=1$, i.e. $|q(q+2)|=1-|q+1|^{2}$, is realized when $\operatorname{Im} q=0$. Therefore, a "window resonance" in $\sigma_{n}(E)$ appears for negative $\operatorname{Re} q$ when (i) $|\operatorname{Im} q| \ll|\operatorname{Re} q|$ and (ii) $|q+1|<1$. (For example, $\operatorname{Re} q=-0.612$, $\operatorname{Im} q=-0.207$ and $\Delta=1.124$ for the results in figure $2(\mathrm{~d})$.)

As is clear from our general considerations, the continuum resonance phenomena shown in Figures 1 and
2 disappear in any theory that does not account for the influence of the atomic potential $U(r)$ on the scattering states (such as, e.g., the Born approximation or even an improved SFA $[1,7])$. However, an accurate non-perturbative account of the interaction of a recombining electron with both a laser field and an atomic potential leads to complicated results even for a potential $U(r)$ supporting only a single bound state, as in the TDER theory (cf the result (25)). Nevertheless, the results simplify for a low-frequency $\left(\hbar \omega \ll\left|E_{0}\right|\right)$ field $\mathbf{F}(t)$, in which case the system (14) can be solved iteratively, taking into account nondiagonal matrix elements $\mathcal{M}_{k, k^{\prime}}(\epsilon)$ perturbatively [21]. In the lowest approximation, neglecting nondiagonal matrix elements, the coefficients $f_{k}(\mathbf{p})$ take the following form:

$$
f_{k}(\mathbf{p}) \approx f_{k}^{(1)}(\mathbf{p})=\frac{c_{k}(\mathbf{p})}{\mathcal{M}_{k, k}(\epsilon)}=\frac{c_{k}(\mathbf{p})}{\mathcal{M}_{0,0}(\epsilon+k \hbar \omega)} .
$$

Also, as noted above, the coefficients $g_{k \neq 0}$ are small for low frequencies, so that we can make the approximation $g_{k} \approx C_{k} \delta_{k, 0}$. As is seen in figure 2, the approximations (34) and $g_{k}=C_{k} \delta_{k, 0}$ reasonably describe the resonant phenomena. The resonant structures originate from the matrix element in the denominator of (34), which has zeros in the complex plane of $\epsilon$ at $\epsilon=\tilde{\varepsilon}-k \hbar \omega$, where $\tilde{\varepsilon}$ is the complex quasienergy $\varepsilon$ in the low-frequency approximation [19].

Finally, we note that resonant phenomena in LARA/ LARR processes cannot be described in the low-frequency Kroll-Watson approximation (KWA) for the scattering state wavefunction [22]. Within the TDER theory, the KWA is formulated in terms of the function $f_{\epsilon}$ $(\mathbf{p}, t)$ in $(12)[23]:$

$$
f_{\epsilon}^{\mathrm{KWA}}(\mathbf{p}, t)=\frac{\mathrm{e}^{-\mathrm{i} \mathcal{S}(\mathbf{p}, t) / \hbar}}{R(\mathcal{E}(t))}, \quad \mathcal{E}(t)=\frac{\mathbf{P}^{2}(t)}{2 m},
$$

where $R(\mathcal{E})$ (cf (17)) is related to the partial s-wave amplitude $f_{0}(E)$ of elastic electron scattering from the potential $U(r)$ in the TDER theory at $\mathbf{F}(t)=0: f_{0}(E)=[\kappa R(E)]^{-1}$ [19]. The shortcoming of the KWA is that in this semiclassical approximation, the quantization of the photon energy is completely neglected so that both $\mathcal{S}(\mathbf{p}, t)$ 
and $R(\mathcal{E}(t))$ in (35) depend only on the classical energy $\mathcal{E}(t)$ of an electron in a laser field, which is always positive. Therefore, although the amplitude $f_{0}(E)$ has a pole at negative energy $E=E_{0}$ (since $R\left(E_{0}\right)=0$ [19]), the function $R(\mathcal{E}(t))$ in (35) has no zeros, i.e. the KWA result (35) fails to describe resonant effects (cf Figure 2). (For this reason, the resonant effects disappear also in the KWA for LAES [10]).

In conclusion, we have analyzed the key features of resonant phenomena in LARA/LARR processes that occur for electron energies corresponding to $\mu$-photon laser-stimulated attachment/recombination. For such energies, we find that the spectra of spontaneously emitted photons in the high-energy parts of the LARA/ LARR plateaus coincide with the harmonic generation spectra of the bound systems. Owing to the significant enhancement of resonant cross sections versus non-resonant ones, we expect that our findings should facilitate experimental observation of the resonant modification of radiative electron attachment/recombination in a laser field and the emission of high-order harmonics in laser-assisted collision processes.

Acknowledgments - This work was supported in part by RFBR grant nos 09-02- 00541 and 10-02-00235, and by NSF grant number PHYS-0901673.

\section{References}

[1] Milošević D B and Ehlotzky F 2003 Adv. At. Mol. Opt. Phys. 49373

[2] Shuman E S, Jones R R and Gallagher T F 2008 Phys. Rev. Lett. 101263001

[3] Jaroń A, Kamiński J Z and Ehlotzky F 2000 Phys. Rev. A 61 023404
[4] Kuchiev M Yu and Ostrovsky V N 2000 Phys. Rev. A 61 033414

[5] Kamińskii J Z and Ehlotzky F 2003 J. Mod. Opt. 50621

[6] Jaroń A, Kamiński J Z and Ehlotzky F 2001 Phys. Rev. A 63 055401

[7] Milošević D B and Ehlotzky F 2002 Phys. Rev. A 65042504

[8] Milošević D B and Ehlotzky F 2003 J. Mod. Opt. 50657

[9] Zheltukhin A N, Manakov N L, Flegel A V, and Frolov M V 2011 JETP Lett. 94599

[10] Flegel A V, Frolov M V, Manakov N L and Starace A F 2009 Phys. Rev. Lett. 102103201

[11] Fano U and Cooper J W 1968 Rev. Mod. Phys. 40441

[12] Zel'dovich Ya B 1967 Sov. Phys. - JETP 241006

[13] Manakov N L, Ovsiannikov V D, and Rapoport L P 1986 Phys. Rep. 141319

[14] Potvliege R and Shakeshaft R 1992 Atoms in Intense Laser Fields ed M Gavrila (New York: Academic) p 373

[15] Manakov N L, Frolov M V, Starace A F and Fabrikant I I 2000 J. Phys. B: At. Mol. Opt. Phys. 33 R141

[16] Frolov M V, Flegel A V, Manakov N L, and Starace A F 2007 Phys. Rev. A 75063407

[17] Manakov N L and Fainshtein A G 1981 Theor. Math. Phys. 48815

[18] Manakov N L, Starace A F, Flegel A V, and Frolov M V 2008 JETP Lett. 8792

[19] Frolov M V, Manakov N L, and Starace A F 2008 Phys. Rev. A 78063418

[20] Frolov M V, Flegel A V, Manakov N L, and Starace A F 2007 Phys. Rev. A 75063408

[21] Manakov N L, Starace A F, Flegel A V, and Frolov M V 2002 JETP Lett. 76258

[22] Kroll N M and Watson K M 1973 Phys. Rev. A 8804

[23] Flegel A V, Frolov M V, Manakov N L, and Zheltukhin A N 2009 J. Phys. B: At. Mol. Opt. Phys. 42241002 